\title{
A Theory for Electromagnetic Radiation and Coupling
}

\author{
Gaobiao Xiao, Shanghai Jiao Tong University
}

(Minor revisions are made in this version, which are marked in red)

(This theory is developed from my previous work:

1. xiao, gaobiao (2021): A Theory for Analysis of Pulse Electromagnetic Radiation. TechRxiv. Preprint. https://doi.org/10.36227/techrxiv.14054051.v4

2. xiao, gaobiao (2020): Electromagnetic Energy Balance Equations and Poynting Theorem. TechRxiv. Preprint. https://doi.org/10.36227/techrxiv.12555698.v1

3. G. Xiao, C. Xiong, S. Huang, R. Liu and Y. Hu, "A New Perspective on the Reactive Electromagnetic Energies and Q factors of Antennas," in IEEE Access, vol. 8, pp. 173790-173803, 2020, doi: 10.1109/ACCESS.2020.3024888.

4. G. Xiao, Y. Hu and S. Xiang, "Comparison of Five Formulations for Evaluating Q Factors of Antennas," 2020 IEEE MTT-S International Conference on Numerical Electromagnetic and Multiphysics Modeling and Optimization (NEMO), 2020, pp. 1-3, doi: 10.1109/NEMO49486.2020.9343605.

The flaw in the previous version that in the case of Hertzian dipole the reactive magnetic energy does not exactly equal the energy stored in the inductor in the equivalent circuital modal has been fixed. More numerical examples will be included in my formal paper.)

Abstract-A theory for analyzing the radiative and reactive electromagnetic energies of a radiator in vacuum is presented. In vacuum, the radiative electromagnetic energies will depart from their sources and travel to infinity, generating a power flux in the space. However, the reactive electromagnetic energies are bounded to their sources. They appear and disappear almost in the same time with their sources, and their fluctuation also causes a power flux in the space. In the proposed theory, the reactive electromagnetic energies of a radiator are defined by postulating that they have properties similar to the self-energies in the charged particle theory. More importantly, in addition to a main term of source-potential products, the reactive energies contain a special energy term which will continue to exist a short time after the sources disappear. This oscillating energy is related to the electric displacement and the vector potential, and seems to be responsible for energy exchanging between the reactive energy and the radiative energy in the radiation process, performing like the Schott energy term. As the Poynting vector describes the total power flux density related to the total electromagnetic energy, it should include the contributions of the propagation of the radiative energies and the fluctuation of the reactive energies. The mutual electromagnetic couplings between two radiators are also defined in a similar way in which the vector potential plays a central role. The reactive electromagnetic energies can be evaluated with explicit expressions in time domain and frequency domain. The theory is verified with the Hertzian dipole and numerical examples.

Index Terms - Reactive energy, electric energy density, magnetic energy density, radiative energy, Poynting vector

\section{INTRODUCTION}

The electromagnetic radiation problems have been intensively investigated for more than a hundred years. It is a little bit strange that there is still no widely accepted formulation for evaluating the stored reactive energies and Q factors of radiators[1]-[14]. The main difficulty may come from the fact that there is no clear definition in macroscopic electromagnetic theory for the reactive electromagnetic energy. It is commonly known in classical charged particle theory that the fields associated with charged particles can be divided into self-fields and radiative fields[15][16]. The self-fields include the Coulomb fields and the velocity fields, carrying self-energies, part of it also referred to as Schott energy in some literatures[17]-[19]. The radiative fields are generated by the acceleration of charged particles, emitting radiative energies to the surrounding space. The self-fields/self-energies are considered to be attached to the charged particles, or simply speaking, they appear with the charged particles and disappear with the charged particles. On the contrary, after being radiated by the charged particles, the radiative fields/energies will depart from the sources and propagate to the remote infinity. They exist after their generation sources disappeared and can couple with other sources they encountered in their journey. Although it is natural to consider that the reactive energies in macroscopic electromagnetics is similar to the self-energies or the Schott energy, no successful attempt has been found or well accepted to handle the reactive energies in this manner. No expressions for reactive energies are established in macroscopic electromagnetics that can be derived rigorously from the self-fields of charged particles.

On the other hand, Poynting vector is widely considered as the electromagnetic power flux density[20]. Poynting 
Theorem describes the relationship between the Poynting vector, the varying rate of the total electromagnetic energy densities, and the work rate done by the exciting source. It provides an intuitive description of the propagation of the electromagnetic energy. However, interpreting the Poynting vector as the electromagnetic power flux density has always been controversial [21]-[31], and some researchers have pointed out that Poynting Theorem may have not been used in the correct way in some situations[32][33]. This difficulty is largely due to the fact that it is not easy to separate from the Poynting vector the true radiative power flux.

It is known that the Poynting Theorem is not convenient to use for evaluating the reactive energies stored by radiators in an open space [5][13], which has been investigated for decades. For harmonic fields, the total electromagnetic energy obtained by integrating the conventional energy densities of $(0.5 \mathbf{D} \cdot \mathbf{E})$ and $(0.5 \mathbf{B} \cdot \mathbf{H})$ over the infinite three-dimensional volume is infinite because they account for the total energy consisting of the radiative energy and the reactive energy. For harmonic fields over the time interval $(-\infty<t<\infty)$, the radiative energy occupies the whole space and is infinitely large [14]. Some researchers suggested that those fields associated with the propagating waves should not contribute to the stored reactive energies. The reactive energies can be made finite by subtracting from the total energy density an additional term associated with the radiative power. However, it is not easy to give a general expression for that term because the propagation patterns are quite different for different radiators [1][5].

Based on these observations, the macroscopic electromagnetic radiation issue is revisited and a new energy/power balance equation at a certain instant time is proposed, which may give an intuitive and reasonable demonstration that the Poynting vector does not only contain the radiative power flux density but also a pseudo power flux caused by the fluctuation of the reactive energies.

It is not the aim of this paper to argue that the reactive energies in the macroscopic electromagnetics are exactly the selfenergy or the Schott energy in the classical charged particles. Instead, a definition for the reactive electromagnetic energies is proposed based on the hypothesis that the reactive energies in the macroscopic electromagnetics bear the same characteristics as the self-energies or Schott energy: (1) they are attached to the sources. They will disappear after their sources disappeared; (2) the definition is in consistent with the stored energies associated with static charges and steady state currents; (3) the reactive energies do not propagate like the radiative energies, but their fluctuation may propagate at the light velocity in vacuum just like the radiative fields. A theory is proposed based on these considerations, in which the radiative energies and the reactive energies can be separated. A special energy term is included in the reactive energy, which performs like the Schott energy in charged particle theory [18]. As a consequence, the Poynting vector is divided into two vectors. One vector mainly accounts for the power flux density associated with the radiative energies and the other vector accounts for the fluctuation of the reactive energies. The theory also provides a simple way to define the mutual electromagnetic couplings between two radiators. One radiator may exert electromagnetic couplings to other sources through its potentials instead of fields.

\section{FORMULATIONS FOR REACTIVE AND RADIATIVE ENERGIES}

For the sake of convenience, we define the energy term associated with a charge density and a current density in free space as follows, respectively,

$$
\begin{aligned}
& W_{\rho}(t)=\int_{V_{s}} \frac{1}{2} \rho\left(\mathbf{r}_{1}, t\right) \phi\left(\mathbf{r}_{1}, t\right) d \mathbf{r}_{1}=\int_{V_{s}} w_{\rho}\left(\mathbf{r}_{1}, t\right) \mathbf{r}_{1} \\
& W_{J}(t)=\int_{V_{s}} \frac{1}{2} \mathbf{A}\left(\mathbf{r}_{1}, t\right) \cdot \mathbf{J}\left(\mathbf{r}_{1}, t\right) d \mathbf{r}_{1}=\int_{V_{s}} w_{J}\left(\mathbf{r}_{1}, t\right) d \mathbf{r}_{1}
\end{aligned}
$$

where the scalar potential $\phi$ and the vector potential $\mathbf{A}$ evaluated at the observation point $\mathbf{r}$ and the time $t$ are defined in their usual way,

$$
\begin{aligned}
& \phi(\mathbf{r}, t)=\int_{V_{s}} \frac{\rho\left(\mathbf{r}_{1}, t^{\prime}\right)}{4 \pi \varepsilon_{0} R_{1}} d \mathbf{r}_{1} \\
& \mathbf{A}(\mathbf{r}, t)=\mu_{0} \int_{V_{s}} \frac{\mathbf{J}\left(\mathbf{r}_{1}, t^{\prime}\right)}{4 \pi R_{1}} d \mathbf{r}_{1}
\end{aligned}
$$

In the above equations, $\rho\left(\mathbf{r}_{1}, t^{\prime}\right)$ and $\mathbf{J}\left(\mathbf{r}_{1}, t^{\prime}\right)$ are the charge density and current density at source point $\mathbf{r}_{1} \in V_{s}$ and retarded time $t^{\prime}=t-R_{1} / c$, in which $c$ is the light velocity in vacuum and $R_{1}=\left|\mathbf{r}-\mathbf{r}_{1}\right|$ is the distance between the two positions. $\mu_{0}$ and $\varepsilon_{0}$ are respectively the permeability and permittivity in free space. The potentials have to satisfy the Lorentz Gauge, and their reference zero points are put at the infinity.

From Maxwell equation, we can obtain the following relationships, 


$$
\begin{gathered}
\frac{1}{2} \mathbf{D} \cdot \mathbf{E}=\frac{1}{2} \phi \rho-\frac{1}{2} \nabla \cdot(\phi \mathbf{D})-\frac{1}{2} \mathbf{D} \cdot \frac{\partial \mathbf{A}}{\partial t} \\
\frac{1}{2} \mathbf{B} \cdot \mathbf{H}=\frac{1}{2} \mathbf{A} \cdot \mathbf{J}+\frac{1}{2} \mathbf{A} \cdot \frac{\partial \mathbf{D}}{\partial t}+\frac{1}{2} \nabla \cdot(\mathbf{A} \times \mathbf{H})
\end{gathered}
$$

where $\mathbf{E}, \mathbf{H}$ and $\mathbf{D}, \mathbf{B}$ are the electromagnetic fields/flux density associated with the sources. Integrating (5) over a domain $V_{a} \supset V_{s}$ and making use of (1) gives

$$
W_{\rho}(t)=\int_{V_{a}}\left(\frac{1}{2} \mathbf{D} \cdot \mathbf{E}+\frac{1}{2} \mathbf{D} \cdot \frac{\partial \mathbf{A}}{\partial t}\right) d \mathbf{r}_{1}+\oint_{S_{a}} \frac{1}{2} \phi \mathbf{D} \cdot \hat{\mathbf{n}} d S
$$

where $S_{a}$ is the surface enclosing $V_{a}$ with outward normal unit $\hat{\mathbf{n}}$. (7) shows that $W_{\rho}(t)$ can be separated into two parts, one part is stored in the domain $V_{a}$, the other part will pass through $S_{a}$ and be stored in the region outside $V_{a}$. Especially, recalling that $\lim _{r \rightarrow \infty}(\mathbf{D} \cdot \hat{\mathbf{r}}) \sim O\left(1 / r^{2}\right), \lim _{r \rightarrow \infty} \phi \sim O(1 / r)$, where $\hat{\mathbf{r}}$ is the unit radial vector, the surface integral at the RHS of (7) approaches zero at $S_{\infty}$ with $r \rightarrow \infty$. Therefore, the electric energy defined by $W_{\rho}(t)$ really has the sense of being stored in the space with no energy leaking to the infinity. Furthermore, it can be checked that $W_{\rho}(t)$ satisfies the three terms listed in the previously specified hypothesis. Therefore, it is reasonable to define the reactive electric energy of the radiator as

$$
W_{\text {react }}^{e}(t)=\int_{V_{\infty}}\left(\frac{1}{2} \mathbf{D} \cdot \mathbf{E}+\frac{1}{2} \mathbf{D} \cdot \frac{\partial \mathbf{A}}{\partial t}\right) d \mathbf{r}_{1}=W_{\rho}(t) \triangleq W_{\text {tot }}^{e}(t)-W_{\text {rad }}^{e}(t)
$$

where $W_{t o t}^{e}(t)=\int_{V_{\infty}}(0.5 \mathbf{D} \cdot \mathbf{E}) d \mathbf{r}_{1}$ denotes the total electric energy, and $W_{\text {rad }}^{e}(t)=\int_{V_{\infty}}(-0.5 \mathbf{D} \cdot \partial \mathbf{A} / \partial t) d \mathbf{r}_{1}$ denotes the total radiative electric energy. It's important to emphasize that because $W_{\text {react }}^{e}(t)=W_{\rho}(t)$ holds for every time instant $t$, the reactive electric energy $W_{\text {react }}^{e}(t)$ become zero when the charge source disappears. In other words, after the charge source decay away completely, there may still exist electromagnetic fields in the space, but the volume integral of (8) is zero.

Integrating (6) over the domain $V_{a} \supset V_{s}$ and making use of (2) gives

$$
W_{J}(t)=\int_{V_{a}}\left(\frac{1}{2} \mathbf{B} \cdot \mathbf{H}-\frac{1}{2} \mathbf{A} \cdot \frac{\partial \mathbf{D}}{\partial t}\right) d \mathbf{r}_{1}+\oint_{S_{a}}\left(\frac{1}{2} \mathbf{H} \times \mathbf{A}\right) \cdot \hat{\mathbf{n}} d S
$$

However, it is not proper to define $W_{J}(t)$ directly as the reactive magnetic energy. Firstly, the surface integral in (9) is usually a bounded but nonzero value at $S_{\infty}$ since $\lim _{r \rightarrow \infty}(\mathbf{H} \times \mathbf{A}) \cdot \hat{\mathbf{r}} \sim O\left(1 / r^{2}\right)$. Therefore, $W_{J}(t)$ is not an energy purely stored in the whole space $V_{\infty}$ because it contains a part of energy that always flows towards the infinity, relating to the electromagnetic radiation. Secondly, in vacuum, the total radiative electric energy of a radiator should equal its total radiative magnetic energy. Denote the total magnetic energy as $W_{t o t}^{m}(t)=\int_{V_{a}}(0.5 \mathbf{B} \cdot \mathbf{H}) d \mathbf{r}_{1}$. If $W_{J}(t)$ is defined as the reactive magnetic energy, it can be checked from (9) that the radiative magnetic energy does not equal the corresponding radiative electric energy $W_{r a d}^{e}(t)$. Thirdly, as has been verified in our previous works[14][34][35], in the case of the Hertzian dipole, the reactive electric energy defined by $W_{\text {react }}^{e}(t)$ is exactly in agreement with the electric energy stored in the capacitor in its equivalent circuit model proposed by Chu [36]. However, the reactive magnetic energy calculated with $W_{J}(t)$ does not exactly equal to the magnetic energy stored in the equivalent inductor. Only their time average values are equal. Taking into account of these factors, the definition of the reactive magnetic energy of a radiator is modified by making the total radiative magnetic energy equal the total radiative electric energy. Explicitly, we define

$$
W_{\text {react }}^{m}(t)=W_{\text {tot }}^{m}(t)-W_{\text {rad }}^{m}(t)=\int_{V_{\infty}}\left(\frac{1}{2} \mathbf{B} \cdot \mathbf{H}+\frac{1}{2} \mathbf{D} \cdot \frac{\partial \mathbf{A}}{\partial t}\right) d \mathbf{r}_{1}
$$

wherein $W_{\text {rad }}^{m}(t)=W_{\text {rad }}^{e}(t)$. Making use of (6), the reactive magnetic energy can be expressed by

$$
W_{\text {react }}^{m}(t)=\int_{V_{s}}\left(\frac{1}{2} \mathbf{A} \cdot \mathbf{J}\right) d \mathbf{r}_{1}+\int_{V_{\infty}} \frac{1}{2} \frac{\partial}{\partial t}(\mathbf{D} \cdot \mathbf{A}) d \mathbf{r}_{1}-\oint_{S_{\infty}}\left(\frac{1}{2} \mathbf{A} \times \mathbf{H}\right) \cdot \hat{\mathbf{n}} d S
$$

Apparently, an additional term, the second volume integral on the RHS, is used to balance the radiative magnetic energy and the radiative electric energy. The leakage energy to infinity contained in $W_{J}(t)$ is now accounted by the surface integral on the RHS. For pulse radiators, the surface integral vanishes because their waves never reach the infinity. It is 
clear that $W_{J}(t)$ appears/disappears with the current source $\mathbf{J}(\mathbf{r}, t)$. In the following, we will show that the second volume integral on the RHS will also disappear not simultaneously but soon after its sources disappeared.

The electric flux density can be expressed by

$$
\begin{aligned}
& \mathbf{D}(\mathbf{r}, t)=-\varepsilon_{0} \nabla \phi(\mathbf{r}, t)-\varepsilon_{0} \frac{\partial}{\partial t} \mathbf{A}(\mathbf{r}, t) \\
& =-\int_{V_{s}} \int_{-\infty}^{\infty} \rho\left(\mathbf{r}_{1}, t_{1}\right) \nabla G\left(t-t_{1}-R_{1} / c\right) d t_{1} d \mathbf{r}_{1}-\frac{1}{c^{2}} \int_{V_{s}} \int_{-\infty}^{\infty} \mathbf{J}\left(\mathbf{r}_{1}, t_{1}\right) \dot{G}\left(t-t_{1}-R_{1} / c\right) d t_{1} d \mathbf{r}_{1}
\end{aligned}
$$

where the superscript "." means derivative with respect to time. The time domain Green's function can be expressed with the Dirac delta function,

$$
G_{1}\left(\mathbf{r}, \mathbf{r}_{1} ; t-R_{1} / c\right)=\frac{\delta\left(t-R_{1} / c\right)}{4 \pi R_{1}}
$$

Denote the second volume integral in the RHS of (10) as $W_{A D}(t)$. Substituting (4) and (12) into it yields integrations over source region

$$
\begin{aligned}
& W_{A D}(t)=\int_{V_{\infty}} \frac{1}{2} \frac{\partial}{\partial t}(\mathbf{D} \cdot \mathbf{A}) d \mathbf{r} \\
& =\int_{V_{\infty}}\left\{\mu_{0} \int_{V_{s}}\left[-\int_{V_{s}} \int_{-\infty}^{\infty} \rho\left(\mathbf{r}_{1}, t_{1}\right) \nabla G_{1} d t_{1} d \mathbf{r}_{1}-\frac{1}{c^{2}} \int_{V_{s}} \int_{-\infty}^{\infty} \mathbf{J}\left(\mathbf{r}_{1}, t_{1}\right) \dot{G}_{1} d t_{1} d \mathbf{r}_{1}\right] \cdot \int_{-\infty}^{\infty} \mathbf{J}\left(\mathbf{r}_{2}, t_{2}\right) G_{2} d t_{2} d \mathbf{r}_{2}\right\} d \mathbf{r}
\end{aligned}
$$

where $G_{1,2}=G\left(t-t_{1,2}-R_{1,2} / c\right)$ and $R_{1,2}=\left|\mathbf{r}-\mathbf{r}_{1,2}\right|$. With the derivations detailed in the Appendix, the integral can be explicitly expressed by an integration of the source distributions,

$$
\begin{aligned}
W_{A D}(t) & =\int_{V_{\infty}} \frac{1}{2} \frac{\partial}{\partial t}(\mathbf{D} \cdot \mathbf{A}) d \mathbf{r}_{1} \\
& =-\frac{1}{8 \pi \varepsilon_{0}} \int_{V_{s}} \int_{V_{s}} \frac{1}{r_{21}}\left[\int_{t-r_{21} / c}^{t} \rho\left(\mathbf{r}_{1}, \tau\right) \dot{\rho}\left(\mathbf{r}_{2}, 2 t-\tau-r_{21} / c\right)+c^{-2} \dot{J}\left(\mathbf{r}_{1}, 2 t-\tau-r_{21} / c\right) \cdot \mathbf{J}\left(\mathbf{r}_{2}, \tau\right) d \tau\right] d \mathbf{r}_{2} d \mathbf{r}_{1}
\end{aligned}
$$

where $r_{21}=\left|\mathbf{r}_{2}-\mathbf{r}_{1}\right|$. Note that $\rho\left(\mathbf{r}_{1}, t_{1}\right) / \mathbf{J}\left(\mathbf{r}_{1}, t_{1}\right)$ and $\rho\left(\mathbf{r}_{2}, t_{2}\right) / \mathbf{J}\left(\mathbf{r}_{2}, t_{2}\right)$ stand for the charge/current sources at $\left(\mathbf{r}_{1}, t_{1}\right)$ and $\left(\mathbf{r}_{2}, t_{2}\right)$, respectively. They are the same function related to the same source distribution. For a pulse source in $[0, T]$, it can be checked that the integral becomes zero when $t \geq T+r_{21, \max } / 2 c$, which reveals that after the sources disappeared, although $\partial[\mathbf{A}(\mathbf{r}, t) \cdot \mathbf{D}(\mathbf{r}, t)] / \partial t$ is not zero everywhere in the space, its volume integral over the whole space, i.e., $W_{A D}(t)$, will soon become zero. In the espresions, $r_{21, \max }$ is the largest distance between two source positions.

The reactive electromagnetic energy is the sum of the reactive electric energy and the reactive magnetic energy,

$$
W_{\text {react }}(t)=\int_{V_{\infty}}\left(\frac{1}{2} \mathbf{D} \cdot \mathbf{E}+\frac{1}{2} \mathbf{B} \cdot \mathbf{H}+\mathbf{D} \cdot \frac{\partial \mathbf{A}}{\partial t}\right) d \mathbf{r}_{1}
$$

or numerically, it is equal to

$$
W_{\text {react }}(t)=\int_{V_{s}}\left(\frac{1}{2} \mathbf{A} \cdot \mathbf{J}+\frac{1}{2} \phi \rho\right) d \mathbf{r}_{1}+\int_{V_{\infty}} \frac{1}{2} \frac{\partial}{\partial t}(\mathbf{D} \cdot \mathbf{A}) d \mathbf{r}_{1}=W_{\rho J}(t)+W_{A D}(t)
$$

The total radiative energy is the sum of the radiative electric energy and the radiative magnetic energy, which is

$$
W_{r a d}(t)=W_{r a d}^{e}(t)+W_{r a d}^{m}(t)=-\int_{V_{\infty}}\left(\mathbf{D} \cdot \frac{\partial \mathbf{A}}{\partial t}\right) d \mathbf{r}_{1}
$$

For static electromagnetic fields, the radiative energy is zero, and the reactive electric (magnetic) energy is exactly the stored electric(magnetic) energy associated with the static charge sources (the steady state current sources).

Denote

$$
W_{\text {rad } 0}(t)=\int_{V_{\infty}} \frac{1}{2}\left(\mathbf{A} \cdot \frac{\partial \mathbf{D}}{\partial t}-\mathbf{D} \cdot \frac{\partial \mathbf{A}}{\partial t}\right) d \mathbf{r}_{1}
$$

The radiative energy can then be divided into 


$$
W_{\text {rad }}(t)=W_{\text {rad } 0}(t)-W_{A D}(t)
$$

With these definitions, the total electromagnetic energy can be expressed with

$$
W_{\text {tot }}(t)=W_{\text {tot }}^{e}(t)+W_{\text {tot }}^{m}(t)=W_{\text {rad }}(t)+W_{\text {react }}(t)=W_{\text {rad } 0}(t)+W_{\rho J}(t)
$$

As shown in the Appendix, $W_{\text {rad } 0}(t)$ can be evaluated with an integration over the source region,

$$
W_{\text {rad } 0}(t)=\frac{1}{8 \pi \varepsilon_{0}} \int_{V_{s}} \int_{V_{s}} \frac{1}{r_{21}}\left\{\begin{array}{l}
\int_{r_{21} / c}^{t}\left[\dot{\rho}\left(\mathbf{r}_{1}, \tau\right) \rho\left(\mathbf{r}_{2}, \tau-r_{21} / c\right)-\dot{\rho}\left(\mathbf{r}_{1}, \tau-r_{21} / c\right) \rho\left(\mathbf{r}_{2}, \tau\right)\right] d \tau \\
+c^{-2} \int_{r_{21} / c}^{t}\left[\mathbf{J}\left(\mathbf{r}_{1}, \tau\right) \dot{J}\left(\mathbf{r}_{2}, \tau-r_{21} / c\right)-\mathbf{J}\left(\mathbf{r}_{1}, \tau-r_{21} / c\right) \dot{J}\left(\mathbf{r}_{2}, \tau\right)\right] d \tau
\end{array}\right\} d \mathbf{r}_{2} d \mathbf{r}_{1}
$$

It can be checked that for a pulse source over $[0, T], W_{\text {rad } 0}(t)=W_{\text {rad } 0}(T)$ for $t \geq T$. However, as seen from (20), the total radiative energy will continue to vary in a small time period $\left[T, T+r_{21, \max } / 2 c\right]$ due to the effect of $W_{A D}(t)$.

Now we may give an interpretation to the radiation process of a pulse radiator with sources existing in $[0, T]$. The reactive electromagnetic energy generated by the pulse radiator includes two parts, as shown in (17). The first part is $W_{\rho J}(t)$. It is strictly attached to the sources and appears/disappears simultaneously with the source. However, the second part, $W_{A D}(t)$, does not disappear simultaneously with its source. It may still remain nonzero within the period of $\left[T, T+r_{21, \max } / 2 c\right]$, i.e., will disappear shortly after the source died away. Since the sources disappeared for $t \geq T$, the nonzero reactive energy $W_{A D}(t)$ cannot be absorbed in vacuum as there is no sources for $t \geq T$. Because of energy conservation, the only possible way is that $W_{A D}(t)$ all converts to radiative energy, corresponding to the change of the radiative energy in the same time interval after the source disappeared. If we assume that the increasing rate of the total radiative energy in this stage equals the decreasing rate of $W_{A D}(t)$, then we can get the relationship expressed in (20). Although I know the argument may become exhausting, it is still proposed here to relate $W_{\text {rad } 0}(t)$ to the radiative rate and consider $W_{A D}(t)$ as something like the Schott energy observed in papers concerning charged particles [17]-[19]. As is discussed in [19], the radiation rate is always nonnegative and it describes an irreversible loss of energy, while the Schott energy changes reversibly. Judging from (17) and (20), it is reasonable to consider that $W_{A D}(t)$ plays a kind of role of exchanging energies between the reactive energies and the radiative energies. On the other hand, for $t \geq T+r_{21 \text { max }} / 2 c$, although $W_{A D}(t)=0$, its integrand is not necessary to be zero everywhere. It causes an energy fluctuation propagating with the radiative fields and contributes to the Poynting vector.

The Poynting Theorem correctly describes the relationship between the work rate done by the source, the total electromagnetic energy in region $V_{a} \supseteq V_{s}$ containing the source, and the total electromagnetic power flux crossing the boundary $S_{a}$ of the region,

$$
-\int_{V_{s}} \mathbf{E} \cdot \mathbf{J} d \mathbf{r}_{1}=\frac{\partial}{\partial t} \int_{V_{a}}\left(\frac{1}{2} \mathbf{D} \cdot \mathbf{E}+\frac{1}{2} \mathbf{B} \cdot \mathbf{H}\right) d \mathbf{r}_{1}+\oint_{S_{a}} \mathbf{S} \cdot \hat{\mathbf{n}} d S
$$

where the Poynting vector $\mathbf{S}=\mathbf{E} \times \mathbf{H}$ is conventionally regarded as the power flux density, like in the antenna society. With the definition of (16) and (18), it can be rewritten as

$$
-\int_{V_{s}} \mathbf{E} \cdot \mathbf{J} d \mathbf{r}_{1}=\frac{\partial}{\partial t} \int_{V_{a}}\left(\frac{1}{2} \mathbf{D} \cdot \mathbf{E}+\frac{1}{2} \mathbf{B} \cdot \mathbf{H}+\mathbf{D} \cdot \frac{\partial \mathbf{A}}{\partial t}\right) d \mathbf{r}_{1}+\int_{V_{a}}\left(-\mathbf{D} \cdot \frac{\partial \mathbf{A}}{\partial t}\right) d \mathbf{r}_{1}+\oint_{S_{a}} \mathbf{S} \cdot \hat{\mathbf{n}} d S
$$

which implies that the Poynting vector contains the contribution from the propagation of the radiative energy and the fluctuation of the reactive energy.

Now we will show that the radiative energy part $W_{\text {rad } 0}(t)$ associated with a bounded volume is a convenient quantity for engineering application. Substituting (5) and (6) into (23) and reorganizing it gives

$$
-\int_{V_{s}} \mathbf{E} \cdot \mathbf{J} d \mathbf{r}^{\prime}-\frac{\partial}{\partial t} W_{\rho J}(t)=\frac{\partial}{\partial t} \int_{V_{a}} \frac{1}{2}\left(\mathbf{A} \cdot \frac{\partial \mathbf{D}}{\partial t}-\mathbf{D} \cdot \frac{\partial \mathbf{A}}{\partial t}\right) d \mathbf{r}_{1}+\oint_{S_{a}}\left[\mathbf{E} \times \mathbf{H}+\frac{1}{2} \frac{\partial}{\partial t}(\mathbf{A} \times \mathbf{H}-\phi \mathbf{D})\right] \cdot \hat{\mathbf{n}} d S
$$

For the sake of convenience, a new vector is introduced for the integrand of the surface integral in (25),

$$
\mathbf{S}_{\text {rad } 0}(\mathbf{r}, t)=\mathbf{E} \times \mathbf{H}-\frac{\partial}{\partial t}\left(\frac{1}{2} \mathbf{H} \times \mathbf{A}+\frac{1}{2} \phi \mathbf{D}\right)
$$

It has to be noted that $\mathbf{S}_{\mathrm{rad} 0}$ is not the radiative power density. Denote its surface integration as 


$$
P_{\text {Srad }}(t)=\oint_{S_{a}} \mathbf{S}_{\text {rad } 0} \cdot \hat{\mathbf{n}} d S
$$

The total work done by the source is

$$
W_{e x c}(t)=-\int_{-\infty}^{t} \int_{V_{s}} \mathbf{E}\left(\mathbf{r}^{\prime}, \tau\right) \cdot \mathbf{J}\left(\mathbf{r}^{\prime}, \tau\right) d \mathbf{r}^{\prime} d \tau
$$

Integrating both side of (25) gives

$$
W_{e x c}(t)-W_{\rho J}(t)=W_{r a d 0}(t)+\int_{0}^{t} P_{r a d 0}(\tau) d \tau
$$

where $W_{\text {rad } 0}(t)$ is defined also with (19) but with the integration domain replaced by $V_{a}$. Accordingly, $P_{\text {Srad }}(t)$ can be interpreted as the part passing through the observation surface $S_{a}$. Since it is not easy to find an explicit expression for the total radiative power passing the observation surface, $P_{\text {Srad }}(t)$ can provide a very good measurement for it. As shown in the Hertzian dipole and the other examples, $P_{S r a d}(t)$ gives a kind of time average value of the total radiative power passing through the observation surface. (29) indicates that it is more convenient to use $W_{\text {rad } 0}(t)$ instead of $W_{\text {rad }}(t)$ in many practical situations.

For $t \geq T+r_{21, \max } / 2 c, \quad W_{\rho J}(t)=W_{A D}(t)=0$, the total radiative energy can be expressed with integration of $P_{S r a d}(t)$ on an arbitrary observation surface enclosing the radiator,

$$
W_{\text {rad }}(t)=W_{\text {rad } 0}(t)=\int_{t_{\min }}^{t_{\max }} P_{\text {Srad }}(t) d t=W_{\text {exc }}(T)
$$

For pulse sources, $P_{\text {Srad }}(t)$ has nonzero values over period $\left(t_{\min }<t<t_{\max }\right)$, in which $t_{\min }$ and $t_{\max }$ correspond respectively to the earliest and the latest time that the fields pass through the observation surface. In a special case, we may choose $V_{a}=V_{s}$, and put the observation surface $S_{a}$ directly on the surface of the sources. Assume that all radiative fields come out of $S_{a}$ will no longer interact with the sources. Ignoring the radiative energy stored in $V_{s}$, we may obtain the power coming out of the surface of the sources as

$$
P_{\text {Srad } 0}(t)=-\int_{V_{s}}\left[\mathbf{E} \cdot \mathbf{J}+\frac{\partial}{\partial t}\left(\frac{1}{2} \rho \phi+\frac{1}{2} \mathbf{A} \cdot \mathbf{J}\right)\right] d \mathbf{r}^{\prime}
$$

Apparently, $P_{\text {Srad }}(t)$ at different observation surfaces are not expected to be equal, but their integrations over the time interval $\left(t_{\min }<t<t_{\max }\right)$ are equal, including that of $P_{\text {Srad } 0}(t)$, since all the radiative energy of the pulse source in vacuum will eventually pass through the observation surface and propagate to infinity.

\section{Mutual COUPLingS}

Assume that two radiators have sources of $\left(\mathbf{J}_{1}, \rho_{1}\right)$ and $\left(\mathbf{J}_{2}, \rho_{2}\right)$ in $V_{s 1}$ and $V_{s 2}$ in vacuum, respectively. The coupling electromagnetic energy from source- 2 to source- 1 is defined as

$$
\begin{aligned}
& W_{12}(t)=\int_{V_{s 1}}\left(\frac{1}{2} \rho_{1} \phi_{2}+\frac{1}{2} \mathbf{J}_{1} \cdot \mathbf{A}_{2}+\frac{1}{2} \frac{\partial}{\partial t}\left(\mathbf{D}_{1} \cdot \mathbf{A}_{2}\right)\right) d \mathbf{r}_{1} \\
& =\frac{1}{8 \pi \varepsilon_{0}} \int_{V_{s 1}} \int_{V_{s 2}} \frac{1}{r_{21}}\left\{\begin{array}{l}
\rho_{1}\left(\mathbf{r}_{1}, t\right) \rho_{2}\left(\mathbf{r}_{2}, t-r_{21} / c\right)+c^{-2} \mathbf{J}_{1}\left(\mathbf{r}_{1}, t\right) \cdot \mathbf{J}_{2}\left(\mathbf{r}_{2}, t-r_{21} / c\right) \\
-\int_{t-r_{21} / c}^{t}\left[\rho_{1}\left(\mathbf{r}_{1}, \tau\right) \dot{\rho}_{2}\left(\mathbf{r}_{2}, 2 t-\tau-r_{21} / c\right)+c^{-2} \mathbf{J}_{1}\left(\mathbf{r}_{1}, 2 t-\tau-r_{21} / c\right) \cdot \mathbf{J}_{2}\left(\mathbf{r}_{2}, \tau\right)\right] d \tau
\end{array}\right\} d \mathbf{r}_{2} d \mathbf{r}_{1}
\end{aligned}
$$

and for that from source-1 to source-2,

$$
\begin{aligned}
& W_{21}(t)=\int_{V_{s 2}}\left(\frac{1}{2} \rho_{2} \phi_{1}+\frac{1}{2} \mathbf{J}_{2} \cdot \mathbf{A}_{1}+\frac{1}{2} \frac{\partial}{\partial t}\left(\mathbf{D}_{2} \cdot \mathbf{A}_{1}\right)\right) d \mathbf{r}_{2} \\
& =\frac{1}{8 \pi \varepsilon_{0}} \int_{V_{s 2}} \int_{V_{s 1}} \frac{1}{r_{21}}\left\{\begin{array}{l}
\rho_{2}\left(\mathbf{r}_{2}, t\right) \rho_{1}\left(\mathbf{r}_{1}, t-r_{21} / c\right)+c^{-2} \mathbf{J}_{2}\left(\mathbf{r}_{2}, t\right) \cdot \mathbf{J}_{1}\left(\mathbf{r}_{1}, t-r_{21} / c\right) \\
-\int_{t-r_{21} / c}^{t}\left[\rho_{2}\left(\mathbf{r}_{2}, \tau\right) \dot{\rho}_{1}\left(\mathbf{r}_{1}, 2 t-\tau-r_{21} / c\right)+c^{-2} \dot{J}_{2}\left(\mathbf{r}_{2}, 2 t-\tau-r_{21} / c\right) \cdot \mathbf{J}_{1}\left(\mathbf{r}_{1}, \tau\right)\right] d \tau
\end{array}\right\} d \mathbf{r}_{1} d \mathbf{r}_{2}
\end{aligned}
$$


where $\mathbf{A}_{1,2}$ and $\phi_{1,2}$ are respectively the vector potentials and scalar potentials by the sources $\left(\rho_{1,2}, \mathbf{J}_{1,2}\right)$. The total reactive energy of the system is then $W_{\text {react tot }}(t)=W_{11}(t)+W_{12}(t)+W_{21}(t)+W_{22}(t)$.

In this theory, the mutual coupling energies also include a Schott energy like term, which may be denoted by $W_{A_{1} D_{2}}(t)$ and $W_{A_{2} D_{1}}(t)$. It is proposed in this theory that the mutual electromagnetic coupling occurs when the potentials from one source propagate to other sources and interact with them or their nearby fields. The mutual coupling is exerted through potentials instead of fields, so the electric and magnetic Aharonov-Bohm effect [38][39] may be interpreted with the classical electromagnetic theory in a simple way, as the change of the mutual coupling energies will inevitably introduce a force on the sources influenced by the mutual coupling.

\section{RADIATION OF HARMONIC SOURCES}

For harmonic fields with time convention of $e^{j \omega t}$, the radiation is assumed to last temporally from $-\infty$ to $+\infty$, so the radiative energy is infinitely large. The Poynting theorem can be applied to describe the balance between the time average powers and the varying rate of the energies,

$$
-\frac{1}{2} \int_{V_{s}} \mathbf{E} \cdot \mathbf{J}^{*} d \mathbf{r}^{\prime}=2 j \omega \int_{V_{a}}\left[\frac{1}{4} \mathbf{B} \cdot \mathbf{H}^{*}-\frac{1}{4} \mathbf{E} \cdot \mathbf{D}^{*}\right] d \mathbf{r}^{\prime}+\frac{1}{2} \oint_{S_{a}} \mathbf{E} \times \mathbf{H}^{*} \cdot \hat{\mathbf{n}} d S
$$

The same symbols are used for the corresponding phasors for the sake of convenience. From which the time average radiative power at infinity can be evaluated with source distributions,

$$
\left(P_{r a d}\right)_{a v}=\operatorname{Re}\left\{\frac{1}{2} \oint_{S_{\infty}} \mathbf{E} \times \mathbf{H}^{*} \cdot \hat{\mathbf{n}} d S\right\}=-\operatorname{Re}\left\{\frac{1}{2} \int_{V_{s}} \mathbf{E} \cdot \mathbf{J}^{*} d \mathbf{r}^{\prime}\right\}
$$

However, the evaluation of the reactive energies in conventional formulation requires to subtract the radiative energy from the total energy. Since both the energies are unbounded, all those formulations based on energy subtraction are not quite satisfactory so far.

With the theory proposed here, the power balance can be evaluated within any domain enclosed by an observation surface $S_{a}$ enclosing the source region $V_{s}$,

$$
-\int_{V_{s}} \mathbf{E} \cdot \mathbf{J}^{*} d \mathbf{r}^{\prime}=2 j \omega \int_{V_{s}}\left(\frac{1}{4} \phi \rho^{*}+\frac{1}{4} \mathbf{A} \cdot \mathbf{J}^{*}\right) d \mathbf{r}^{\prime}+\oint_{S_{a}}\left[\frac{1}{2} \mathbf{E} \times \mathbf{H}^{*}-j \omega\left(\frac{1}{4} \mathbf{H}^{*} \times \mathbf{A}+\frac{1}{4} \phi \mathbf{D}^{*}\right)\right] \cdot \hat{\mathbf{n}} d S
$$

The time average radiative power crossing the observation surface can be obtained using the radiative power flux vector $\mathbf{S}_{\text {Srad }}$ or the source distributions,

$$
\left(P_{r a d}\right)_{a v}=\operatorname{Re}\left\{\oint_{S_{a}}\left[\frac{1}{2} \mathbf{E} \times \mathbf{H}^{*}-\frac{1}{4} j \omega\left(\mathbf{H}^{*} \times \mathbf{A}+\phi \mathbf{D}^{*}\right)\right] \cdot \hat{\mathbf{n}} d S\right\}=-\operatorname{Re}\left\{\int_{V_{s}} \frac{1}{2} \mathbf{E}\left(\mathbf{r}^{\prime}\right) \cdot \mathbf{J}^{*}\left(\mathbf{r}^{\prime}\right) d \mathbf{r}^{\prime}\right\}
$$

Note that the observation surface is not required to approach infinity for evaluating the radiative power. It can be checked that the result is in consistent with that obtained using the Poynting vector, as has been shown in [14] that

$$
\operatorname{Re}\left\{\oint_{S_{\infty}} j \omega\left(\frac{1}{4} \mathbf{H}^{*} \times \mathbf{A}+\frac{1}{4} \phi \mathbf{D}^{*}\right) \cdot \hat{\mathbf{n}} d S\right\}=0
$$

The average reactive energy can be calculated with the fields and the vector potential,

$$
\left(W_{\text {react }}\right)_{a v}=\operatorname{Re}\left\{\int_{V_{\infty}}\left(\frac{1}{4} \mathbf{E} \cdot \mathbf{D}^{*}+\frac{1}{4} \mathbf{B} \cdot \mathbf{H}^{*}+\frac{1}{2} j \omega \mathbf{A} \cdot \mathbf{D}^{*}\right)\right\}
$$

It is straightforward to check that $\left(W_{A D}\right)_{a v}=0$, so the time average reactive energy can be alternatively calculated using the source-potential products as

$$
\left(W_{\text {react }}\right)_{a v}=\operatorname{Re}\left\{\int_{V_{s}}\left(\frac{1}{4} \rho \phi^{*}+\frac{1}{4} \mathbf{A} \cdot \mathbf{J}^{*}\right) d \mathbf{r}^{\prime}\right\}
$$

It can be easily shown that the average value of $W_{A_{2} D_{1}}(t)$ and $W_{A_{1} D_{2}}(t)$ are also zero. The time average mutual coupling electromagnetic energies are then

$$
\left\{\begin{array}{l}
\left(W_{12}\right)_{a v}=\operatorname{Re} \int_{V_{s 1}}\left(\frac{1}{4} \rho_{1} \phi_{2}^{*}+\frac{1}{4} \mathbf{J}_{1}^{*} \cdot \mathbf{A}_{2}\right) d \mathbf{r}_{1} \\
\left(W_{21}\right)_{a v}=\operatorname{Re} \int_{V_{s 2}}\left(\frac{1}{4} \rho_{2} \phi_{1}^{*}+\frac{1}{4} \mathbf{J}_{2}^{*} \cdot \mathbf{A}_{1}\right) d \mathbf{r}_{2}
\end{array}\right.
$$


As expected, $\left(W_{21}\right)_{a v}=\left(W_{12}\right)_{a v}$ holds for mutual coupling in free space.

\section{HertZian Dipole}

A Hertzian dipole locating at the origin is analyzed to show the energy/power balance relationship. The moment of the dipole is assumed to be $q l \cos \omega t$, the scalar potential and the vector potential of which can be readily derived from the Hertzian potential $\Pi=(q l / 4 \pi r) \cos (\omega t-k r) \quad$ [34][40],

$$
\begin{aligned}
& \mathbf{A}=-\frac{\omega \mu_{0} q l}{4 \pi r} \sin (\omega t-k r)(\hat{\mathbf{r}} \cos \theta-\hat{\boldsymbol{\theta}} \sin \theta) \\
& \varphi=\frac{\omega^{2} \mu_{0} q l}{4 \pi} \cos \theta\left[\frac{1}{k^{2} r^{2}} \cos (\omega t-k r)-\frac{1}{k r} \sin (\omega t-k r)\right]
\end{aligned}
$$

from which the fields are found to be

$$
\begin{gathered}
\mathbf{E}=\frac{k^{2} q l}{4 \pi \varepsilon_{0} r}\left\{\hat{\mathbf{r}} 2 \cos \theta\left[\frac{1}{k^{2} r^{2}} \cos (\omega t-k r)-\frac{1}{k r} \sin (\omega t-k r)\right]+\hat{\boldsymbol{\theta}} \sin \theta\left[\left(\frac{1}{k^{2} r^{2}}-1\right) \cos (\omega t-k r)-\frac{1}{k r} \sin (\omega t-k r)\right]\right\} \\
\mathbf{H}=-\frac{\omega k q l}{4 \pi r} \sin \theta\left[\frac{1}{k r} \sin (\omega t-k r)+\cos (\omega t-k r)\right] \hat{\boldsymbol{\varphi}}
\end{gathered}
$$

As is known, the Hertzian dipole is a point source and its total reactive energy is infinite. A common strategy is to evaluate the integrals (8) and (10) in the whole space excluding a small sphere with radius $a$. The results are found to be

$$
\begin{gathered}
W_{\text {react }}^{e}(t)=\int_{V_{\infty}-V_{a}}\left(\frac{1}{2} \mathbf{D} \cdot \mathbf{E}+\frac{1}{2} \mathbf{D} \cdot \frac{\partial \mathbf{A}}{\partial t}\right) d \mathbf{r}^{\prime}=\alpha_{0}\left[\frac{1}{k^{3} a^{3}}+\frac{1}{k a}+\left(\frac{1}{k^{3} a^{3}}-\frac{1}{k a}\right) \cos 2(\omega t-k a)-\frac{2}{k^{2} a^{2}} \sin 2(\omega t-k a)\right] \\
W_{\text {react }}^{m}(t)=\int_{V_{\infty}-V_{a}}\left(\frac{1}{2} \mathbf{B} \cdot \mathbf{H}+\frac{1}{2} \mathbf{D} \cdot \frac{\partial \mathbf{A}}{\partial t}\right) d \mathbf{r}^{\prime}=\frac{2 \alpha_{0}}{k a} \sin ^{2}(\omega t-k a)
\end{gathered}
$$

The radiative power evaluated at a spherical observation surface is

$$
P_{\text {Srad }}(t)=\oint_{S_{a}} \mathbf{S}_{\text {Srad }} \cdot \hat{\mathbf{n}} d S=2 \omega \alpha_{0}
$$

It is a constant value independent of the radius of the sphere, clearly indicating that the total radiative power associated with $W_{\text {rad } 0}(t)$ crossing any concentric spherical surface is the same.

For comparison, the surface integral of the Poynting vector on the spherical surface $S_{a}$ is

$$
P_{p v}(t)=\oint_{S_{a}} \mathbf{S} \cdot \hat{\mathbf{n}} d S=2 \omega \alpha_{0}\left[1+\left(\frac{2}{k a}-\frac{1}{k^{3} a^{3}}\right) \sin 2(\omega t-k a)+\left(1-\frac{2}{k^{2} a^{2}}\right) \cos 2(\omega t-k a)\right]
$$

which varies with the radius of the surface. As expected, the time average of $P_{p v}(t)$ equals that of $P_{\text {Srad }}(t)$. The time average energies are listed below,

$$
\left\{\begin{array}{l}
\left(W_{m}\right)_{a v}=\alpha_{0}\left(\frac{1}{k a}\right) \\
\left(W_{e}\right)_{a v}=\alpha_{0}\left(\frac{1}{k^{3} a^{3}}+\frac{1}{k a}\right)
\end{array}\right.
$$

The Q factor of the dipole is then calculated to be

$$
Q=\frac{2 \omega\left(W_{e}\right)_{a v}}{\left(P_{r a d}\right)_{a v}}=\frac{1}{k^{3} a^{3}}+\frac{1}{k a}
$$

which is exactly in agreement with the result shown in [41].

The well-established equivalent circuit model proposed by Chu [36] for Hertzian dipole is shown in Fig.1. Assuming that the current in the radiation resistor at the interface of $r=a$ is $i_{R}=I_{0} \cos (\omega t-k a)$, the energies stored in the capacitor and the inductor can be derived to be

$$
\left\{\begin{array}{l}
W_{C}(t)=\frac{I_{0}^{2}}{4 \omega}\left[\frac{1}{k a}+\frac{1}{k^{3} a^{3}}+\left(\frac{1}{k^{3} a^{3}}-\frac{1}{k a}\right) \cos 2(\omega t-k a)-\frac{2}{(k a)^{2}} \sin 2(\omega t-k a)\right] \\
W_{L}(t)=\frac{I_{0}^{2}}{2 \omega}\left(\frac{1}{k a} \sin ^{2}(\omega t-k a)\right)
\end{array}\right.
$$




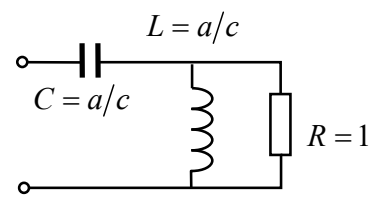

Fig.1 Equivalent circuit model for Hertzian dipole radiation.

If we choose $I_{0}^{2}=4 \omega \alpha_{0}$, it can be verified that $W_{C}(t)=W_{e}(t)$, and $W_{L}(t)=W_{m}(t)$. This exact agreement gives a good support to the proposed theory.

The integration regions for $W_{\text {rad }}(t), W_{\text {rad } 0}(t)$ and $W_{A D}(t)$ are all modified in a similar way. They are found to be

$$
\begin{gathered}
W_{r a d}(t)=-\int_{V_{\infty}-V_{a}} \mathbf{D} \cdot \frac{\partial \mathbf{A}}{\partial t} d V=2 k \alpha_{0} \lim _{r \rightarrow \infty}(r-a)+\alpha_{0}\left[\sin 2(\omega t-k a)-\lim _{r \rightarrow \infty} \sin 2(\omega t-k r)\right] \\
W_{\text {rad } 0}(t)=\int_{V_{\infty}-V_{a}} \frac{1}{2}\left(\mathbf{A} \cdot \frac{\partial \mathbf{D}}{\partial t}-\mathbf{D} \cdot \frac{\partial \mathbf{A}}{\partial t}\right) d V=2 \alpha_{0} k \lim _{r \rightarrow \infty}(r-a) \\
W_{A D}(t)=\int_{V_{\infty}-V_{a}} \frac{\partial}{\partial t}\left(\frac{1}{2} \mathbf{A} \cdot \mathbf{D}\right) d V=-\alpha_{0}\left[\sin 2(\omega t-k a)-\lim _{r \rightarrow \infty} \sin 2(\omega t-k r)\right]
\end{gathered}
$$

With the wave travels to infinity, the total radiative energy $W_{r a d 0}(t)$ monotonically increases with the radius, revealing that the radiative rate is always positive. The Schott term like energy $W_{A D}(t)$ oscillates in the propagation with a zero average value. Its amplitude remains constant in this case.

\section{Short Pulse Radiators}

The radiation of a solenoidal loop current is analyzed again, with the numerical results of $W_{A D}(t)$ added. The solenoidal surface current on a ring is described by $\mathbf{J}_{s}(\mathbf{r}, t)=\mathbf{f}(\mathbf{r}) I(t)[\mathrm{A} / \mathrm{m}]$, as shown in Fig.2. Here we choose

$$
\mathbf{f}(\mathbf{r})=1.0 \hat{\varphi}
$$

The inner and outer radius of the ring is $0.08 \mathrm{~m}$ and $0.1 \mathrm{~m}$, respectively. The temporal function is a modulated Gaussian pulse,

$$
I(t)= \begin{cases}e^{-\gamma^{2}} \sin \omega t, & 0 \leq t \leq T \\ 0, & \text { else }\end{cases}
$$

with $\omega=2 \pi \times 10^{10}, \gamma=2 \sqrt{5}(t-0.5 T) / T, T=1 \mathrm{~ns}$. Therefore, both its initial and final reactive energy are zero. Two spherical surfaces with radius of $0.2 \mathrm{~m}$ and $10 \mathrm{~m}$ chosen are as observation surfaces, with their centers coinciding with that of the source. They are labeled by sphere- 1 and sphere-2, respectively. The radiative energies $W_{\text {rad } 0}(t)$ passing through sphere-1, 2 are calculated with integration of $P_{\text {Srad }}(t)$, as expressed in (30). $W_{p v}(t)$ is the integration of the Poynting vector power passing through the observation surfaces,

(Notes: the results in the previous preprint https://doi.org/10.36227/techrxiv.14054051.v4 have to be corrected because the currents are doubled in program by my coding miss.)

$$
W_{p v}(t)=\int_{0}^{t} P_{S p v}(\tau) d \tau=\int_{0}^{t} \oint_{s p h e r e-1,2} \mathbf{S}\left(\mathbf{r}_{1}, \tau\right) \cdot \hat{\mathbf{n}} d \mathbf{r}_{1} d \tau
$$

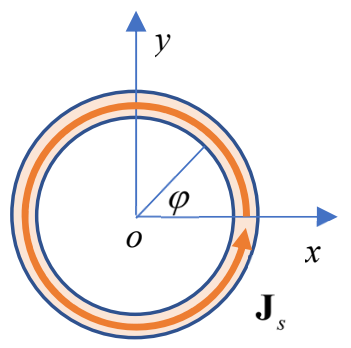

Fig. 2 Solenoidal loop current.

The excitation energy, radiative energy $W_{\text {rad } 0}(t)$ and the energy evaluated with Poynting vector are shown in Fig. 3(a). In this case, the reactive energy includes the contribution from the current alone since the corresponding charge is zero, so 
it is denoted as $W_{J}$ in the figures. $W_{J}$ oscillates with the source and admits negative values periodically. In the proposed theory, it is acceptable because the reactive energy is dependent on the potentials, which are values relative to their reference zero points. When the current varies and changes its direction periodically, the retarded vector potential in the resource region lags behind and may point in direction opposite to that of the current, causing negative values. The energy $W_{A D}(t)$ is also plotted in Fig.3(a), and is zoomed in in Fig.3(b) together with $W_{J}$. It is shown that $W_{A D}(t)$ oscillates like $W_{J}$, but continue to exist for about $0.33 \mathrm{~ns}$ after the source disappeared at $1 \mathrm{~ns}$. Note that the Schott energy in the charged particle theory may also be negative [19][42].

The energies passing through sphere-1 are shown in Fig. 3(c). The smallest and the largest distance between the source and sphere- 1 are respectively $0.1 \mathrm{~m}$ and $0.3 \mathrm{~m}$. The total radiative energy passed at $t=2 \mathrm{~ns}$ is equal to that evaluated at the source region.

The excitation power, radiative power $P_{\text {Srad }}$ and the time varying rate of the reactive energy are shown in Fig. 4(a). The powers crossing-1 and sphere-2 are shown in Fig.4(b) and (c), respectively. The radiative power $P_{\text {Srad }}(t)$ varies smoothly and remains positive. The Poynting power contains ripples coming from the fluctuation of $P_{\text {Sreact }}(t)$, which gradually decrease with the propagation distance.

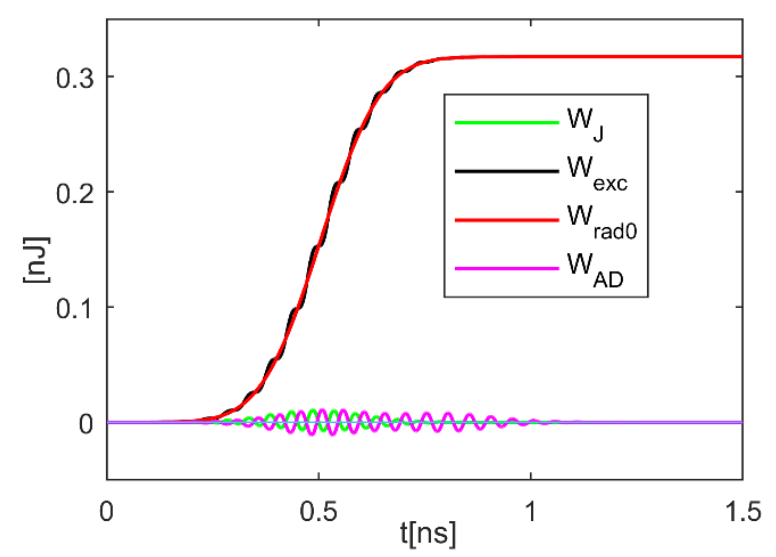

(a)

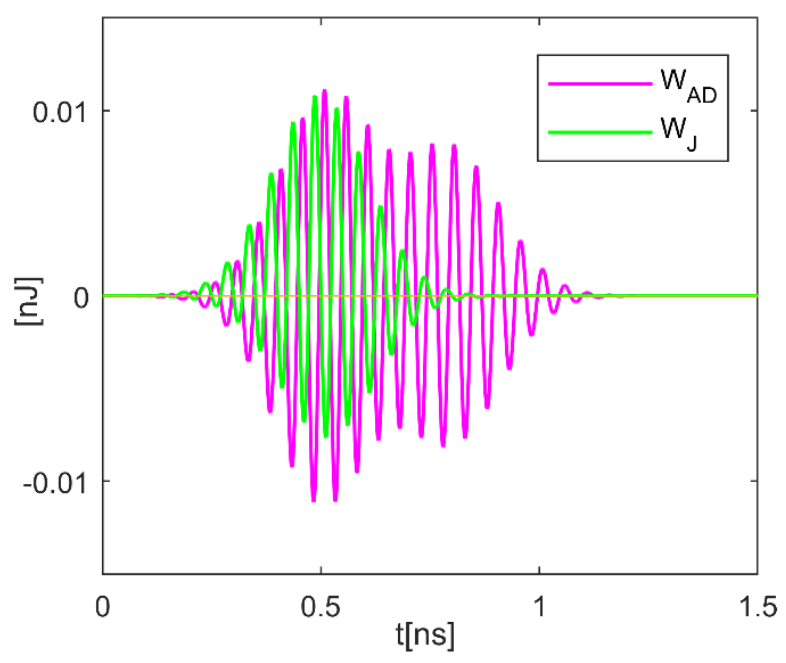

(b) 


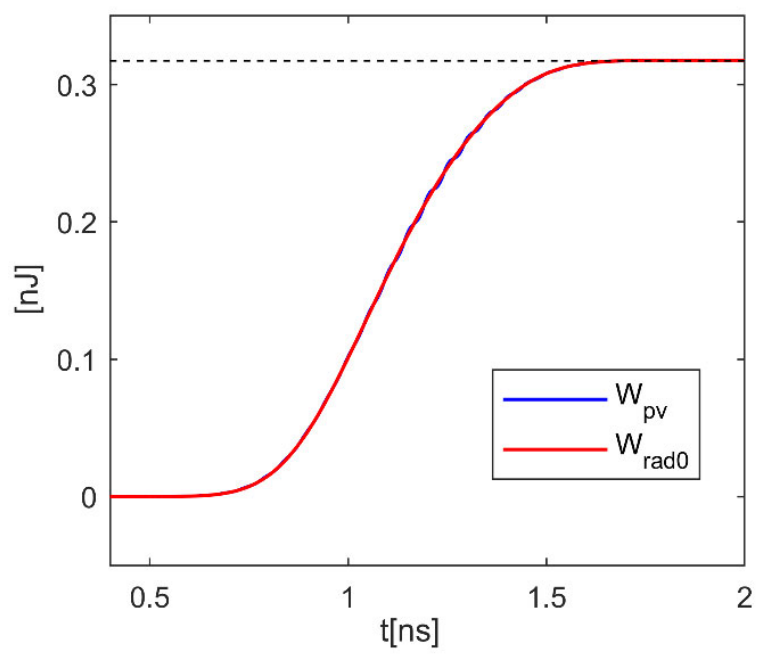

(c)

Fig.3 The energies of the loop current. (a) The total excitation energy, reactive energy, radiative energy and $W_{A D}$ evaluated in the source region. (b) The zoomed in figure for $W_{J}$ and $W_{A D}$. (c) The energies crossing sphere-1.

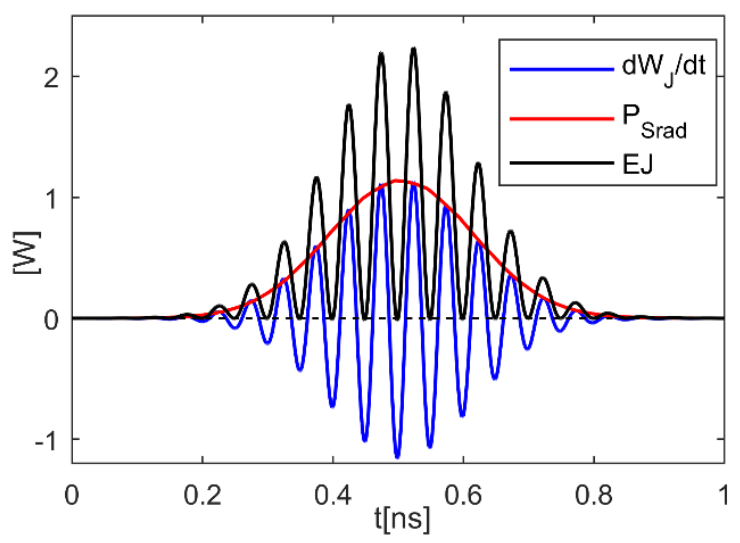

(a)

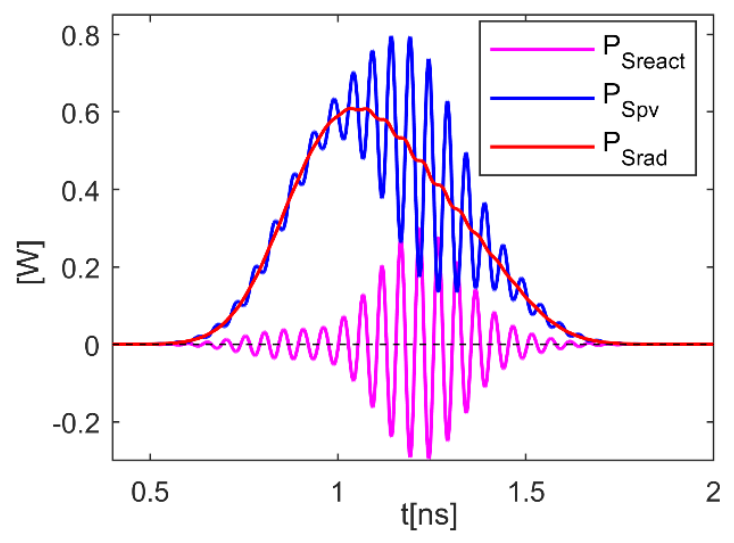

(b) 


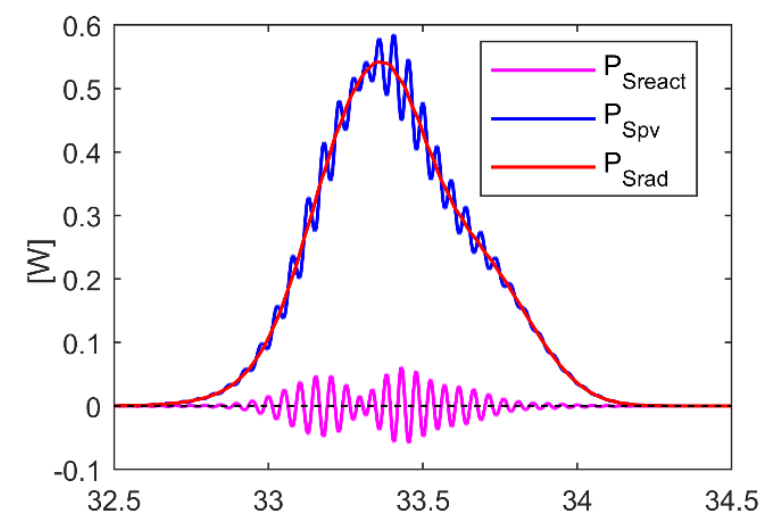

(c)

Fig.4 The powers of the loop current. (a) The excitation power, radiative power and the varying rate of the reactive energy evaluated in the source region. (b) The powers crossing sphere-1. (c) The powers crossing sphere-2.

\section{CONCLUSIONS}

Some issues concerning with the electromagnetic radiation and mutual couplings remain confusing or even controversial for decades long, especially the definitions for the reactive energy and Q factors of radiators. This theory proposes clear definitions and explicit expressions for the reactive energy and the radiative energy of a radiator. The introduction of a Schott energy like term in the reactive energy makes it possible to separate the radiative energy and the reactive energy in a reasonable manner. Consequently, a new power balance equation is given by modifying the Poynting relation so that the Poynting vector is divided into two parts, accounting for the contribution from the radiative energy propagation and the fluctuation of the reactive energy. The newly defined reactive energy term, $W_{\operatorname{rad} 0}(t)$, and its flux, $P_{\text {Srad }}(t)$, can characterize the radiative energy almost satisfactorily. Furthermore, they can be numerically evaluated more efficiently, so are the mutual electromagnetic coupling energies defined with potentials.

Although in the theory, expressions for the reactive electric energy and the reactive magnetic energy are also separately provided, it is strongly recommended to combine the two reactive energies together and treating them as a whole.

The theory is different from Carpenter formulation [23], in which it was proposed to use the source-potential combination terms in (16) as the total electromagnetic energy, and to replace the Poynting Theorem with a new equation. The formulation, as well as the power flow vector $\phi \vec{J}$ by Slepian [43], was pointed to be mathematically flawed by Dr. Endean [44]. In the theory proposed here, the source-potential terms are considered to form the reactive energy together with a Schott energy like term. They compose only part of the total electromagnetic energy. So the theory does not suffer from the mathematical flaws checked by Dr. Endean since there is no modification to the total electromagnetic energy and the correspondent Poynting Theorem.

\section{Appendix}

(15) and (22) can be obtained using the method given in [6], involving the evaluation of the following key integration associated with two source point $\mathbf{r}_{1}$ and $\mathbf{r}_{2}$,

$$
I=\int_{V_{\infty}} G\left(\tau_{1}-R_{1} / c\right) G\left(\tau_{2}-R_{2} / c\right) d \mathbf{r}=\frac{c^{2}}{16 \pi^{2}} \int_{V_{\infty}} \frac{1}{R_{1} R_{2}} \delta\left(c \tau_{1}-R_{1}\right) \delta\left(c \tau_{2}-R_{2}\right) d \mathbf{r}
$$

where $\tau_{1,2}=t-t_{1,2}, \quad R_{1,2}=\left|\mathbf{r}-\mathbf{r}_{1,2}\right|$. The value of the integral has been given in the equation (32) in [6]. Here we provide an alternative rigorous proof. In the spherical coordinates, choose $\mathbf{r}_{1}$ as the origin, and put $\mathbf{r}_{2}$ on $+z$ axis. Therefore, we can write $\mathbf{r}_{2}=r_{21} \hat{\mathbf{z}}, R_{1}=|\mathbf{r}|=r, R_{2}=\left|\mathbf{r}-\mathbf{r}_{2}\right|=\sqrt{r^{2}-2 r r_{21} \cos \theta+r_{21}^{2}}$. According to the symmetry of the integrand, we have

$$
\begin{aligned}
& I=\frac{c^{2}}{8 \pi} \int_{0}^{\pi} \int_{0}^{\infty} \frac{1}{r R_{2}} \delta\left(c \tau_{1}-r\right) \delta\left(c \tau_{2}-R_{2}\right) r^{2} \sin \theta d r d \theta=\frac{c^{2}}{8 \pi} \int_{-1}^{1} \frac{c \tau_{1}}{R_{2}} \delta\left(c \tau_{2}-R_{2}\right) d \cos \theta \\
& =-\frac{c^{2}}{8 \pi} \int_{\left|c \tau_{1}-r_{21}\right|}^{c \tau_{1}+r_{21}} \frac{c \tau_{1}}{R_{2}} \delta\left(c \tau_{2}-R_{2}\right)\left(-\frac{R_{2}}{c \tau_{1} r_{21}}\right) d R_{2}=\frac{c^{2}}{8 \pi r_{21}}
\end{aligned}
$$

where $d R_{2}=-c \tau_{1} r_{21} / R_{2} d \cos \theta$ is used. The integration range of $\left(\tau_{1}, \tau_{2}\right)$ for nonzero $I$ is determined by 


$$
\left|c \tau_{1}-r_{21}\right| \leq c \tau_{2} \leq c \tau_{1}+r_{21}
$$

which is exactly the same as eq. (33) in [6].

Next, take the first term of $W_{A D}(t)$ as an example to show the derivation of (15). Rearranging the integration order gives

$$
W_{A D}^{\rho}(t)=-\mu_{0} \frac{1}{2} \frac{\partial}{\partial t} \int_{V_{s}} \int_{V_{s}} \int_{-\infty}^{\infty} \int_{-\infty}^{\infty} \mathbf{J}\left(\mathbf{r}_{1}, t_{1}\right) \rho\left(\mathbf{r}_{2}, t_{2}\right) \cdot \int_{V_{\infty}} G_{1} \nabla G_{2} d \mathbf{r} d t_{2} d t_{1} d \mathbf{r}_{2} d \mathbf{r}_{1}
$$

Making use of the identities $G_{1} \nabla G_{2}=\nabla\left(G_{1} G_{2}\right)-\nabla G_{1} G_{2}, \quad \mathbf{J}_{1} \cdot \nabla G_{1}=-\mathbf{J}_{1} \cdot \nabla_{1} G_{1}=G_{1} \nabla_{1} \cdot \mathbf{J}_{1}-\nabla_{1} \cdot\left(\mathbf{J}_{1} G_{1}\right)$, and ignoring the surface integrals at $S_{\infty}$, we get

$$
\begin{aligned}
& W_{A D}^{\rho}(t)=-\mu_{0} \frac{\partial}{\partial t} \int_{V_{s}} \int_{V_{s}} \int_{-\infty}^{\infty} \int_{-\infty}^{\infty} \nabla_{1} \cdot \mathbf{J}\left(\mathbf{r}_{1}, t_{1}\right) \rho\left(\mathbf{r}_{2}, t_{2}\right) \int_{V_{\infty}} G_{1} G_{2} d \mathbf{r} d t_{2} d t_{1} d \mathbf{r}_{2} d \mathbf{r}_{1} \\
& =\frac{\mu_{0} c^{2}}{8 \pi} \frac{\partial}{\partial t} \int_{V_{s}} \int_{V_{s}} \int_{-\infty}^{\infty} \int_{-\infty}^{\infty} \frac{1}{r_{21}} \nabla_{1} \cdot \mathbf{J}\left(\mathbf{r}_{1}, t_{1}\right) \rho\left(\mathbf{r}_{2}, t_{2}\right) d t_{2} d t_{1} d \mathbf{r}_{2} d \mathbf{r}_{1} \\
& =-\frac{\mu_{0} c^{2}}{16 \pi} \frac{\partial}{\partial t} \int_{V_{s}} \int_{V_{s}} \int_{-\infty}^{\infty} \int_{-\infty}^{\infty} \frac{1}{r_{21}} \dot{\rho}\left(\mathbf{r}_{1}, t_{1}\right) \rho\left(\mathbf{r}_{2}, t_{2}\right) d t_{1} d t_{2} d \mathbf{r}_{2} d \mathbf{r}_{1}
\end{aligned}
$$

Performing the double integration $\left(\iint d t_{1} d t_{2}\right)$ on the region limited by (61), and dividing the inner integration into three sub-regions gives,

$$
\begin{aligned}
& W_{A D}^{\rho}(t)=-\frac{\mu_{0} c^{2}}{16 \pi} \frac{\partial}{\partial t} \int_{V_{s}} \int_{V_{s}} \frac{1}{r_{21}}\left\{\begin{array}{l}
\int_{0}^{r_{21} / c} \rho\left(\mathbf{r}_{2}, t_{1}+r_{21} / c\right) \rho\left(\mathbf{r}_{1}, t_{1}\right) d t_{1} \\
+\int_{r_{21} / c}^{t-r_{21} / c}\left[\rho\left(\mathbf{r}_{2}, t_{1}+r_{21} / c\right)-\rho\left(\mathbf{r}_{2}, t_{1}-r_{21} / c\right)\right] \rho\left(\mathbf{r}_{1}, t_{1}\right) d t_{1} \\
\int_{t-r_{21} / c}^{t}\left[\rho\left(\mathbf{r}_{2}, 2 t-t_{1}-r_{21} / c\right)-\rho\left(\mathbf{r}_{2}, t_{1}-r_{21} / c\right)\right] \rho\left(\mathbf{r}_{1}, t_{1}\right) d t_{1}
\end{array}\right\} d \mathbf{r}_{2} d \mathbf{r}_{1} \\
& =-\frac{\mu_{0} c^{2}}{8 \pi} \int_{V_{s}} \int_{V_{s}} \int_{t-r_{21} / c}^{t} \frac{1}{r_{21}} \rho\left(\mathbf{r}_{1}, t_{1}\right) \dot{\rho}\left(\mathbf{r}_{2}, 2 t-t_{1}-r_{21} / c\right) d t_{1} d \mathbf{r}_{2} d \mathbf{r}_{1}
\end{aligned}
$$

Replacing $t_{1}$ by $\tau$ and evaluating the second term in a similar way we can get (15). The nonzero range can be determined by noting that the sources exist in $[0, T]$ and one of the source terms is zero for $t>T+r_{21, \max } / 2 c$.

\section{References}

[1] R. E. Collin and S. Rothschild, "Evaluation of antenna Q," IEEE Trans. Antennas Propag., vol. AP-12, no. 1, pp. 2327, Jan. 1964.

[2] A. Shlivinski and E. Heyman, "Time-domain near-field analysis of short pulse antennas-Part I: Spherical wave (multipole) expansion," IEEE Trans. Antennas Propag., vol. 47, no. 2, pp. 271-279, Feb. 1999.

[3] A. Shlivinski and E. Heyman, "Time-domain near-field analysis of short pulse antennas-Part II: Reactive energy and the antenna Q," IEEE Trans. Antennas Propag., vol. 47, no. 2, pp. 280-286, Feb. 1999.

[4] A. D. Yaghjian, "Internal energy, Q-energy, Poynting's theorem, and the stress dyadic in dispersive material," IEEE Trans. Antennas Propag., vol. 55, no. 6, pp. 1495-1505, Jun. 2007.

[5] G. A. E. Vandenbosch, "Reactive energies, impedance, and Q factor of radiating structures," IEEE Trans. Antennas Propag., vol. 58, no. 4, pp. 1112-1127, Apr. 2010.

[6] G. A. E. Vandenbosch, "Radiators in time domain-Part I: Electric, magnetic, and radiated energies," IEEE Trans. Antennas Propag., vol. 61, no. 8, pp. 3995-4003, Aug. 2013.

[7] G. A. E. Vandenbosch, "Radiators in time domain -Part II: Finite pulses, sinusoidal regime and Q factor," IEEE Trans. Antennas Propag., vol. 61, no. 8, pp. 4004-4012, Aug. 2013.

[8] M. Capek, L. Jelinek, P. Hazdra, and J. Eichler, "The measurable Q factor and observable energies of radiating structures," IEEE Trans. Antennas Propag., vol. 62, no. 1, pp. 311-318, Jan. 2014.

[9] M. Gustafsson and B. L. G. Jonsson, "Antenna Q and stored energy expressed in the fields, currents, and input impedance," IEEE Trans. Antennas Propag., vol. 63, no. 1, pp. 240-249, Jan. 2015.

[10]W. Geyi, "Stored energies and radiation Q," IEEE Trans. Antennas Propag., vol. 63, no. 2, pp. 636-645, Feb. 2015.

[11]M. Capek, L. Jelinek, and G. A. E. Vandenbosch, "Stored electromagnetic energy and quality factor of radiating structures," Proc. Roy. Soc. A, Math., Phys. Eng. Sci., vol. 472, no. 2188, pp. 20150870, 2016. 
[12]G. A. E. Vandenbosch, "Recoverable energy of radiating structures," IEEE Trans. Antennas Propag., vol. 65, no. 7, pp. 3575-3588, Jul. 2017.

[13]K. Schab et al., "Energy stored by radiating systems," IEEE Access, vol. 6, pp. 10553-10568, 2018.

[14]G. B. Xiao, C. Xiong, S. Huang, R. Liu, Y. Hu, “A new perspective on the reactive electromagnetic energies and Q factors of antennas," IEEE Access, vol. 8, 8999565, pp. 173790-173803, Oct. 2020.

[15]J. D. Jackson, "Classical Electrodynamics;” 3rd ed., John Wiley \& Sons: New York, NY, USA, 1998.

[16]F. Rohrlich, "Classical Charged Particles," $3^{\text {rd }}$ ed., World Scientific Publishing: Singapore, 2007.

[17]T. Nakamura, "On the Schott term in the Lorentz-Abraham-Dirac equation," Quantum Beam Sci., vol. 4, pp. 34, 2020.

[18]G. A. Schott, "Electromagnetic radiation and the mechanical reactions arising from it," Cambridge University Press: Cambridge, UK, 1912.

[19]Ø. Grøn, "The significance of the Schott energy for energy-momentum conservation of a radiating charge obeying the Lorentz-Abraham-Dirac equation,” Am. J. Phys., vol. 79, no. 1, pp. 115-122, 2011.

[20]J. H. Poynting, "On the connexion between electric current and the electric and magnetic inductions in the surrounding field," Proc. Royal Soc. London. vol. 38, pp. 168-172, 1884-1885.

[21]C. S. Lai, “Alternative choice for the energy flow vector of the electromagnetic field," Am. J. Phys., vol. 49, no.9, 841843, Jan. 1981.

[22]R. H. Romer, "Alternatives to the Poynting vector for describing the flow of electromagnetic energy," Am. J. Phys., vol. 50, no.12, pp.1166-1168, Nov. 1982.

[23]C. J. Carpenter, "Electromagnetic energy and power in terms of charges and potentials instead of fields." IEE Proc. $A$, vol. 136, no. 2, pp.55-65, Mar 1989.

[24]L. S. Czarnecki, "Energy flow and power phenomena in electrical circuits: illusions and reality," Electrical Engineering, vol. 82, no.3, pp.119-126, Mar. 2000.

[25]Z. Cakareski, A. E. Emanuel, "Poynting vector and the quality of electric energy," European Trans. Electrical Power, vol. 11 , no. 6, 375-381, Nov. 2001.

[26]A. Chubykaloa, A. Espinozab, and R. Tzonchevc, "Experimental test of the compatibility of the definitions of the electromagnetic energy density and the Poynting vector," European Physical J. D, vol. 31, no.1, pp.113-120, Oct. 2004.

[27]L. S. Czarnecki, “Could power properties of three-phase systems be described in terms of the Poynting vector?” IEEE Trans. Power Delivery, vol. 21, no. 1, pp. 339-344, Jan. 2006.

[28]A. E. Emanuel, "Poynting vector and the physical meaning of nonactive powers," IEEE Trans. Instrument. Measure., vol. 54, no. 4, pp.1457-1462, Aug. 2005.

[29]A.E. Emanuel, “About the rejection of Poynting vector in power systems analysis," J. Electrical Power quality Utilization, vol.8, no.1, pp.43-48, 2007.

[30]P. Kinsler, A. Favaro and M. W. McCall, "Four Poynting theorems," Eur. J. Phys., vol. 30, no. 5, pp. 983-993, Aug. 2009.

[31]J. D. Jackson, "How an antenna launches its input power into radiation: the pattern of the Poynting vector at and near an antenna," Am. J. Phys., vol. 74, no.4, 280-288, Jul. 2005.

[32]A. Kholmetskii, O. Missevitch, T. Yarman, "Poynting Theorem, relativistic transformation of total energy-momentum and electro-magnetic energy-momentum tensor," Found Phys., vol. 46, pp. 236-261, 2016.

[33]A. K. Singal, "Poynting flux in the neighborhood of a point charge in arbitrary motion and radiative power losses," Eur. J. Phys., vol. 37 045210, May 2016.

[34]G. Xiao, "A Theory for Analysis of Pulse Electromagnetic Radiation," TechRxiv. Preprint. https:/doi.org/ 10.36227/techrxiv.14054051.v4, 2021.

[35]G. Xiao, "Electromagnetic Energy Balance Equations and Poynting Theorem," TechRxiv. Preprint. https://doi.org/ 10.36227/techrxiv.12555698.v1,2020.

[36]L. J. Chu, "Physical limitations on omni-directional antennas," J. Appl. Phys., vol. 19, no. 12, pp. 1163-1175, 1948.

[37]Xuezhe Tian, Gaobiao Xiao, and Shang Xiang, "Application of analytical expressions for retarded-time potentials in analyzing the transient scattering by dielectric objects," IEEE Antennas Wireless Propag. Lett., vol.13, pp.1313-1316, 2014.

[38]Y. Aharonov, D. Bohm. "Significance of electromagnetic potentials in quantum mechanics," Phys. Rev., vol. 115, no.3, pp. 485-491, 1959.

[39] Y. Aharonov, D. Bohm. "Further considerations on electromagnetic potentials in the quantum theory," Phys. Rev., vol. 123 , no. 4, pp. 1511-1524, 1961.

[40]J. A. Kong, "Fundamentals in electromagnetic wave theory," 3rd ed., Cambridge, MA, USA: EMW Publishing, 2008, pp.65-67.

[41]J. S. McLean, "A re-examination of the fundamental limits on the radiation Q of electrically small antennas," IEEE Trans. Antennas Propag., vol. 44, no. 5, pp. 672-676, May 1996.

[42] D. R. Rowland, "Physical interpretation of the Schott energy of an accelerating point charge and the question of whether a uniformly accelerating charge radiates," Eur. J. Phys., vol. pp. 31, 1037-1051, Jul. 2010. 
[43]J. Slepian, "Energy and energy flow in the electromagnetic field," J. Appl. Phys. 13, 512-518, 1942; doi: $10.1063 / 1.1714903$

[44]V. G. Endean and C. J. Carpenter, "Electromagnetic energy and power in terms of charges and potentials instead of fields," IEE Proc. A, vol. 139, no. 6, pp. 338-342, Nov. 1992. 